\title{
Fluoroalkyl ether electrolyte with redefined in-cell ion affinity upgrades rate performances of lithium ion batteries
}

\section{Liwei Dong}

Harbin Institute of Technology

\section{Yuanpeng Liu}

Harbin Institute of Technology

\section{Kechun Wen}

Harbin Institute of Technology

\section{Dongjiang Chen}

University of Electronic Science and Technology of China

Jipeng Liu

Harbin Institute of Technology

\section{Botao Yuan}

Harbin Institute of Technology

\section{Yunfa Dong}

Harbin Institute of Technology

\section{Ze Wu}

Harbin Institute of Technology

Yifang Liang

Harbin Institute of Technology

\section{Mengqiu Yang}

Harbin Institute of Technology

Jianyi Ma

Sichuan University

Chunhui Yang

Harbin Institute of Technology

Weidong He ( $\square$ weidong.he@hit.edu.cn )

Harbin Institute of Technology

\section{Article}

Keywords: Long-cycle Rate Performance, Ultra-high Polarity, Solvation-free Li+ Transfer, Uniform 
Posted Date: December 4th, 2020

DOI: https://doi.org/10.21203/rs.3.rs-108440/v1

License: (c) (i) This work is licensed under a Creative Commons Attribution 4.0 International License. Read Full License 


\section{Abstract}

Lithium ion batteries have profound impact on every corner of human life. However, strong solvation of $\mathrm{Li}+$ and rough interfacial ion transfer associated with conventional electrolytes limit the long cycle, highrate battery operation of lithium ion batteries. Herein we design an electrolyte system based on fluoroalkyl ether 2,2,2-trifluoroethyl-1,1,2,3,3,3-hexafluoropropyl ether (THE) and ether electrolytes to effectively upgrade the long-cycle rate performances of electrodes in general. With THE rich in fluoroalkyl groups adjacent to oxygen atoms, the electrolyte owns an ultra-high polarity, enabling solvation-free Li+ transfer with an energy barrier of $0.11 \mathrm{eV}$, only $1 / 7$ of that for conventional ether electrolytes, and 10-times enhancement in $\mathrm{Li}+$ transference at electrolyte/anode interface. In addition, the uniform adsorption of fluorine-rich THE on anode and subsequent LiF formation suppress dendrite formation and stabilize the solid electrolyte interphase layer. With the electrolyte, LiFePO4 cathodes deliver unprecedented cyclic performances with only $0.0012 \%$ capacity loss cycle- 1 over 5000 cycles at $10 \mathrm{C}$, along with upgraded rate performances as observed for other mainstream electrodes including LiCoO2, LiNi0.5Mn0.3Co0.2O2 and Li4Ti5012.

\section{Introduction}

Humans have entered an era featured with information technology, instant communication and convenient transportation, together calling up the extensive applications of rechargeable lithium ion batteries (LIBs). ${ }^{1-3}$ Towards high-capacity LIBs, cathode materials have gone through generation transitions from $\mathrm{LiCoO}_{2}$ (LCO) to $\mathrm{LiFePO}_{5}$ (LFP) and then to $\mathrm{LiNi}_{x} \mathrm{Mn}_{\mathrm{y}} \mathrm{Co}_{1-\mathrm{x}-\mathrm{y}} \mathrm{O}_{2}$ (NCM), and in the meanwhile anodes beyond graphite such as $\mathrm{Li}_{4} \mathrm{Ti}_{5} \mathrm{O}_{12}$ (LTO) have also been commercialized. ${ }^{4-7}$ In addition to the aforementioned commercial electrodes, many other materials have been actively investigated and some have been regarded as promising energy storage materials to meet the requirements of high-capacity, long cycle and high rate LIBs, such as Li air batteries and Li-S batteries among other novel batteries with Li metal anode. ${ }^{8,9}$ Despite the widespread adoption and countless research efforts, there still exist unsatisfactory and even disastrous drawbacks associated with current LIBs, including degrading capacity over cycles, slow charge/discharge rate and safety-related fatalities. These battery properties are all closely correlated with the composition of the electrolyte which transfers $\mathrm{Li}^{+}$between electrodes and forms electrolyte/electrode interfaces. For instance, the electrolyte with excellent wettability increases the contact area with the separator and expedites ion migration to enhance rate capacity. ${ }^{10}$ The compatibility between electrolyte and electrode ensures uniform and reversible $\mathrm{Li}^{+}$ transmission. ${ }^{11}$ Further, the electrolyte directly determines the composition and electrochemical property of solid electrolyte interface (SEI), laying a profound impact on the cycle life and safety of LIBs. ${ }^{12}$ Electrolyte solvents used in LIBs are mainly ethers and carbonates, owing to their wide electrochemical windows and the capability to form stable cathode interface. ${ }^{13}$ However, multiple challenges, including dendrite electrodeposition and parasitic reactions, arise from reactive anodes as well as rough and sluggish electrolyte/electrode interface ion transference of conventional electrolytes. ${ }^{14}$ In addition, these 
hazards. Extensive efforts have been focused on developing powerful new electrolyte systems to address these challenges including: (1) solid-state electrolytes with safety and high-energy-density, such as inorganic solid electrolyte ${ }^{15}$ and solid-state polymer electrolyte; ${ }^{16}$ (2) novel electrolyte solvents with excellent physicochemical properties, such as ionic liquid with excellent ion conductivity, ${ }^{17}$ non-toxic and non-flammable aqueous solvent, ${ }^{18}$ and nitrile with high oxidation potential; ${ }^{19}$ (3) electrolyte additives with SEl formation or flame retardancy, such as lithium difluorobis (oxalato) phosphate (LiDFBOP), ${ }^{20}$ fumed silica, ${ }^{21}$ and trimethyl phosphate, ${ }^{22}(4)$ high concentrated electrolytes with advanced solvation structure. ${ }^{23,24}$ However, solid-state electrolytes with rough electrolyte/electrode interfaces cause excessive interface impedance and result in low ionic conductivity, and novel solvents, additives and highlyconcentrated electrolytes often come with increased viscosity, resulting in poor wettability of separators and the electrodes.

In this report, we develop an electrolyte system based on fluoroalkyl ether 2,2,2-trifluoroethyl-1,1,2,3,3,3hexafluoropropyl ether (THE) and ether electrolytes. The THE electrolyte significantly enhances the interface ion transference to ultimately enhance the in-cell ionic conduction of batteries with prototype conventional electrodes, and effectively suppresses the Li dendrite growth by forming an inorganic LiFrich SEl. With the electrolyte, the $\mathrm{LiFePO}_{4}$ cathode exhibits unprecedented cyclic performances with $0.0012 \%$ capacity loss cycle ${ }^{-1}$ over 5000 cycles at $10 \mathrm{C}$, in addition to the upgraded performances of other typical commercial electrodes including LCO, NCM523 and LTO.

\section{Results And Discussion}

\section{Physicochemical properties of THE electrolyte:}

The compositions of the electrolytes investigated in this work are shown in Table S1 (Supporting Information). THE has outstanding anti-oxidation stability owning to robust electron-withdrawing groups $\left(\mathrm{CF}_{3}\right.$ and $\left.\mathrm{CF}_{2}\right)$. As shown in Figs. 1(a) and 1(e), it exhibits a much lower highest occupied molecular orbital (HOMO) energy value (-8.48 eV) and a much lower lowest unoccupied molecular orbitals (LUMO) level (-0.75 eV) as compared with commercial 1, 3-dioxolane (DOL) and dimethoxyether (DME) solvents, indicating its high-voltage stability and ready reduction on the anode with SEl formation. As shown in Fig. 1(b), conventional DOL + DME electrolyte causes uneven deposition of $\mathrm{Li}^{+}$since rough and sluggish interfacial ion transference between anode and electrolyte produces dead Li on the anode surface, leading to irreversible $\mathrm{Li}^{+}$transmission. After 100 cycles in the DOL + DME electrolyte, a loose layer is observed on the surface of Li anode (Fig. S1a, Supporting Information). This porous layer (Fig. 1C) further aggravates the rough deposition, resulting in continuous corrosion of the Li metal, as evidenced with the low Coulombic efficiency (CE) and poor cycling stability (Figs. 3d-g). The porous dead Li layer with a thickness of $124 \mu \mathrm{m}$ is clearly observed from the cross-sectional scanning electron microscopy (SEM) image (Fig. 1d). This means that the Li metal is severely corroded with the DOL + DME electrolyte, causing the rapid decay of the capacity. ${ }^{25}$ In contrast, for $60 \%$ THE electrolyte, abundant LiF is formed at Loading [MathJax]/jax/output/CommonHTML/fonts/TeX/fontdata.js Ilent ion transmission ability and stability (6.4 V 
$\left.v s \mathrm{Li} / \mathrm{Li}^{+}\right),{ }^{26}$ and enables compact packing in the SEl to isolate the Li metal from the electrolyte. Furthermore, LiF owns high interfacial energy with Li metal, ${ }^{27}$ which accelerates $\mathrm{Li}^{+}$migration along the interface and promotes the parallel growth of Li dendrites along Li metal plane instead of vertical growth. The protective effect of LiF-rich SEI on the Li anode is clearly observed in the SEM images. The anode surface layer in the $60 \%$ THE electrolyte (Figs. $1 \mathrm{~g}$ and S1b, Supporting Information) is much more compactly integrated than that in the DOL + DME electrolyte. Especially, the corrosion of the Li metal is greatly reduced in the $60 \%$ THE electrolyte, and only the top layer $(20 \mu \mathrm{m})$ is corroded after 100 cycles (Fig. 1h). The dense surface layer owns three advantages: (1) reliable security because the ball-like Li morphology is less likely to pierce separator; (2) high CE because the dense layer prevents continuous reaction to reduce the consumption of $\mathrm{Li}$ anode and electrolyte; (3) additional volumetric capacity because dense Li packing reduces the volume. In the elemental mappings of $\mathrm{Li}$ anodes in DOL +DME (Figs. S2a and S2b, Supporting Information) and 60\%THE (Figs. S2c and S2d, Supporting Information) electrolytes after cycling, C elemental mapping is mainly derived from electrolyte solvents and, thus, is selected as the representative of organic species. ${ }^{13}$ The Li metal in the $60 \%$ THE electrolyte contains less C element as compared with that in the DOL + DME electrolyte, implying reduced solvent decomposition in the $60 \% \mathrm{THE}$ electrolyte. F elemental mapping is taken as the representative of the SEI layer. ${ }^{13}$ The Li metal in the $60 \%$ THE electrolyte contains more $F$ element as compared with that in the DOL + DME electrolyte, indicating that the addition of THE is favorable for the formation of the SEl layer. Figures 1(i-k) show X-ray photoelectron spectroscopy (XPS) of the SEl layer in the 60\%THE electrolyte. The organic species formed with the ether electrolyte solvent, including $\mathrm{C}=\mathrm{O}, \mathrm{C}-\mathrm{O}$, and $\mathrm{C}-\mathrm{H} / \mathrm{C}-\mathrm{C}$, are studied with $\mathrm{C} 1 \mathrm{~s}$ spectrum (Fig. 1i). Obvious signals of $\mathrm{CF}_{3}$ and $\mathrm{C}-\mathrm{F}$ are observed, as attributed to the cleavage of the fluorinated groups of THE. As shown in Fig. 1(j), a main peak at $~ 685.7 \mathrm{eV}$ is observed, implying that the $\mathrm{F}$ element in the SEl layer exists mainly in the form of F-Li bond. ${ }^{28}$ The signal of F-Li bond is also observed in Li 1 s XPS spectra, as shown in Fig. 1(k). In-situ Raman spectroscopy is used to study the internal change of THE-based electrolyte during charging and discharging. The test battery configuration is shown in Fig. S3 (Supporting Information). As displayed in Figs. $1(\mathrm{I})$ and $1(\mathrm{~m})$, extensive LiF $(\approx$ $409 \mathrm{~cm}^{-1}$ ) is formed in the $60 \%$ THE electrolyte, considerably above that in the DOL + DME electrolyte, which is attributed to the cleavage of numerous C-F in THE. This is also demonstrated through Fouriertransform infrared (FTIR) spectra of the Li anodes after cycling (Fig. S4, Supporting Information). For the $60 \%$ THE electrolyte, the peak of fluorine-containing species (C-F stretching vibration) at $\sim 1000 \mathrm{~cm}^{-1}$ increases apparently after the first charge ${ }^{29}$ indicating the cleavage of the fluorinated segment of THE molecules. In contrast, there is no obvious fluorine-containing signal in the DOL + DME electrolyte.

Flammable commercial electrolytes exists a safety risk in high-rate operations of lithium ion batteries. ${ }^{30}$ The thermal stability of ethylene carbonate (EC) + dimethyl carbonate (DMC), DOL + DME, and 60\%THE electrolytes is evaluated, as shown in Figs. 2a-C and Videos S1-S3 (Supporting Information). In Videos S1S3, commercial EC + DMC and DOL + DME electrolytes are readily ignited and burned quickly, whereas the $60 \%$ THE electrolyte is non-flammable even with repeated igniting. As the objective leaves the heat source, there are raqing flames in $\mathrm{EC}+\mathrm{DMC}$ and $\mathrm{DOL}+\mathrm{DME}$ electrolytes, but no flame is observed in the $60 \% \mathrm{THE}$ Loading [MathJax]/jax/output/CommonHTML/fonts/TeX/fontdata.js 
electrolyte (Fig. 2c). The ultra-low flammability of THE is attributed to the fluorine substitution at the alkyl moiety of THE, inhibiting the propagation of free oxygen radicals during combustion. The affinity of electrolyte solvents with the polypropylene (PP) separator is studied with density functional theories (DFT). As shown in Fig. 2d, the adsorption energy of THE- $\mathrm{CH}_{2} \mathrm{CHCH}_{3}(-0.12 \mathrm{eV})$ is much below those of DOL- $\mathrm{CH}_{2} \mathrm{CHCH}_{3}(-0.076 \mathrm{eV})$ and $\mathrm{DME}-\mathrm{CH}_{2} \mathrm{CHCH}_{3}(-0.044 \mathrm{eV})$, indicating the outstanding affinity between PP and THE. The $60 \%$ THE electrolyte owns a high wettability with the separator, leading to an increased electrolyte uptake (Figs. 2e and 2f). The viscosity of the $60 \%$ THE electrolyte is only $1.8 \mathrm{mPa} \mathrm{s}$, considerably lower than those of EC + DMC (3.1 mPa s) and DOL + DME (3.5 mPa s) electrolytes. In particular, the $60 \% \mathrm{THE}$ electrolyte maintains the hardly unchanged viscosity at low temperatures (Fig. $2 \mathrm{~g}$ ). The conductivities and $\mathrm{Li}^{+}$transference numbers $\left(\mathrm{t}_{\mathrm{Li}^{+}}\right)$of the electrolytes are displayed in Fig. $2 \mathrm{~h}$. The conductivity of the $60 \% \mathrm{THE}$ electrolyte is slightly lower, but reasonably comparable to those of EC + DMC and DOL + DME electrolytes (that is, $6.2 \mathrm{mS} \mathrm{cm}^{-1}$ versus $10.3 \mathrm{mS} \mathrm{cm}^{-1}$ and $9.2 \mathrm{mS} \mathrm{cm}^{-1}$ ). Different from conductivity, $\mathrm{t}_{\mathrm{Li}}$ increases with increasing THE in the electrolytes from 0.240 for $20 \% \mathrm{THE}, 0.262$ for $40 \% \mathrm{THE}$, and 0.301 for $60 \% \mathrm{THE}$ to 0.310 for $80 \% \mathrm{THE}$, which are all above those of for EC+DMC (0.211) and DOL+DME (0.225). In particualr, as shown in Fig. 2i, compared with commercial electrolytes the $60 \%$ THE electrolyte exhibits a wider electrochemical window and is thus capable of supporting higher voltage battery systems.

\section{Electrochemical behaviors of commercial electrodes with THE electrolyte}

As shown in Fig. S5, the Li/LFP battery with the 60\%THE electrolyte owns the largest capacity and the electrochemical performances of the $60 \% \mathrm{THE}$ electrolyte are investigated. Due to the high ion transmission capability of LiF-rich SEI and excellent separator wettability, the 60\%THE electrolyte greatly increases the rate performance (Figs. 3a-c) and cycling performance (Figs. 3d-g) of the battery. As shown in Figs. 3(a) and S6 (Supporting Information), the LFP cathode with the 60\%THE electrolyte delivers discharge capacities of $153.2 \mathrm{mAh} \mathrm{g}^{-1}, 124.1 \mathrm{mAh} \mathrm{g}^{-1}, 109.8 \mathrm{mAh} \mathrm{g}^{-1}, 95.2 \mathrm{mAh} \mathrm{g}^{-1}, 82.0 \mathrm{mAh} \mathrm{g}^{-1}$, $72.8 \mathrm{mAh} \mathrm{g}^{-1}, 49.8 \mathrm{mAh} \mathrm{g}^{-1}$ and $38.1 \mathrm{mAh} \mathrm{g}^{-1}$ from $1 \mathrm{C}$ to $100 \mathrm{C}$. The capacity recovers to $97.8 \%$ of the initial capacity as the current density is decreased from $100 \mathrm{C}$ back to $1 \mathrm{C}$, indicating the excellent reversibility of the $60 \% \mathrm{THE}$ electrolyte. In contrast, the discharge capacities are considerably lower at all C rates for the EC + DMC electrolyte. For further verification, a high-loading LFP electrode of $13.46 \mathrm{mg} \mathrm{cm}^{-2}$ is utilized. As shown in Fig. 3(d), the battery with the 60\% THE electrolyte maintains a stable cycle at $0.61 \mathrm{~mA} \mathrm{~cm}^{-2}$, while the battery with original electrolyte undergoes decrease in both capacity and $\mathrm{CE}$. The Li/LTO battery using $60 \%$ THE electrolyte also exhibits obvious advantages in electrochemical performances over the commercial electrolyte (Fig. S7, Supporting Information). In addition, the LFP cathode is assembled into full cells with LTO and graphite anodes. Significantly improved cycling and rate performances are achieved in full batteries (Figs. 3b, 3e, and S8, Supporting Information). For other major commercial cathode materials including LCO and NCM532, the batteries with the $60 \%$ THE electrolyte also achieve improved rate and cycling performances as compared with these with the commercial electrolyte (Figs. 3c, 3f, and S9, Supporting Information), indicating that the 60\% THE

Loading [MathJax]/jax/output/CommonHTML/fonts/TeX/fontdata.js t less improvement in performances is observed 
for NCM811 full cells using 60\%THE electrolyte (Fig. S10, Supporting Information), as attributed to the similarity in the radii of $\mathrm{Ni}^{2+}$ and $\mathrm{Li}^{+}$. In the delithiation state, the rapid transfer of $\mathrm{Li}^{+}$in THE-based electrolyte generates a large number of vacant sites as occupied by $\mathrm{Ni}^{2+}$, resulting in an irreversible phase transition of the cathode structure.

The long-cycle, high-rate performances of the Li/LFP cells with EC + DMC and 60\%THE electrolytes are shown in Figs. $3 \mathrm{~g}$ and S11. The cells with the 60\% THE electrolyte give rise to higher capacity and better capacity retention as compared with the EC + DMC electrolyte at all rates (Table S2, Supporting Information). In particular, the battery shows an unprecedented cycle retention with only $0.0012 \%$ capacity loss cycle ${ }^{-1}$ over 5000 cycles at 10 C (Table S3, Supporting Information). The low-temperature performances of the Li/LFP batteries with EC + DMC and 60\% THE electrolytes are studied (Fig. S12, Supporting Information). The battery with the $60 \%$ THE electrolyte also exhibits more pronounced rate capacities as compared with the commercial electrolyte at low temperatures. As displayed in Table S4 (Supporting Information), with the 60\%THE electrolyte, the battery capacities have no significant change at various rates $\left(<5 \mathrm{mAh} \mathrm{g}^{-1}\right)$ at $25^{\circ} \mathrm{C}$ and $0{ }^{\circ} \mathrm{C}$. The outstanding low-temperature performances of the $60 \%$ THE electrolyte are due to the hardly unchanged viscosity with changing temperature (Fig. $2 \mathrm{~g}$ ) and excellent compatibility between electrolyte and separator (Figs. 2d-f), ensuring efficient in-cell ionic conduction. In contrast, the high viscosity of the EC + DMC electrolyte at low temperatures causes slow ion transport and the battery is severely polarized, leading to the low capacity.

\section{Mechanism of rate-performance improvement with THE-based electrolyte}

$A b$ initio molecular dynamics (AIMD) simulations are employed to investigate the solvation structure and rate performances of electrolytes. Fig. S13 shows the simulation snapshots of $E C+D M C, D O L+D M E$ and $60 \%$ THE electrolytes, respectively. The representative configurations of coordinated molecules in the first $\mathrm{Li}^{+}$shell in the three different electrolyte systems are depicted with a ball-and-stick model (Figs. 4a-c). $\mathrm{Li}^{+}$ prefers to coordinate with oxygen from $\mathrm{EC}, \mathrm{DMC}, \mathrm{DOL}$ and DME solvent molecules, facilitating the dissociation of the lithium salts, while THE is a free solvent molecule and does not coordinate with either $\mathrm{Li}^{+}$or anions. Therefore, the dissociation of lithium salts and the number of charge carriers in the THEbased electrolyte decrease with increasing volume ratio of THE, resulting in reduced $\mathrm{Li}^{+}$cations $(0$ and $\mathrm{TFSI}^{-}$anions (). In the $60 \%$ THE electrolyte, the $\mathrm{Li}^{+}$cations are weakly solvated with solvent molecules, and meanwhile the anions are seriously dragged by the $\mathrm{Li}^{+}$cations in return, resulting in a low the mobility of $\mathrm{TFSI}^{-}$anions (). As shown in Eqs. 1 and 2, the ion conductivity and $\mathrm{Li}^{+}$transference number own opposite trends with increasing volume ratio of THE (Fig. 2h). Electrostatic potential (ESP) is calculated to study the impact of electron-withdrawing fluoroalkyl groups on the properties of solvent molecules (Figs. 4d-f). For the DME molecule, the negative potential mainly is concentrated on $\mathrm{O}$ atoms (Fig. 4d), while the THE molecule owns a uniform negative potential distribution in the presence of electron-withdrawing fluoroalkyl groups, demonstrating that THE is unable to coordinate with positivelycharged $\mathrm{Li}^{+}$(Fig. 4e). As the two $\mathrm{F}$ atoms on the carbon adjacent to the $\mathrm{O}$ atom are replaced with $\mathrm{H}$ 
trends with that of DME (Fig. 4f), indicating that the two electron-withdrawing fluoroalkyl groups adjacent to the oxygen atom result in the low solvating capability of lithium ions.

As shown in Tables S5 and S6, the ion conductivity of each component in the battery with DOL + DME and $60 \%$ THE electrolytes is studied. The slowest ion conduction inside the battery occurs at the electrolyte/anode interface and is on the order of $10^{-7} \mathrm{~S} / \mathrm{cm}$, which is far below that of the electrolytes. The ion transport at electrolyte/electrode interface and in the electrodes is contributed by $\mathrm{Li}^{+}$transfer. Therefore, the $\mathrm{Li}^{+}$conduction at the electrolyte/electrode interfaces, instead of the $\mathrm{Li}^{+}$conduction in the electrolyte, is the limiting factor for the rate performances of the battery system. With a smaller ionic conductivity, the THE-based electrolyte owns a larger $\mathrm{Li}^{+}$transference number and enables much enhanced rate performances of the batteries, indicating the THE-based electrolyte greatly enhances the $\mathrm{Li}^{+}$conduction at the electrolyte/electrode interfaces. Due to the extremely low HOMO energy value $(<-8.4 \mathrm{eV})$ and the high oxidation potential $(>5.6 \mathrm{~V})$, THE is free of oxidation on the surface of the cathode (Figs. 1e and S14, Supporting Information). Therefore, the composition of cathode electrolyte interphase (CEI) in the 60\%THE electrolyte is the same as that in commercial electrolyte. As shown in XPS (Figs. 1j and $1 \mathrm{k}$ ) and AIMD (Fig. 4h) results, compared with DOL and DME molecules, abundant LiF forms at the surface of Li metal due to the C-F bond cleavage in THE, resulting in an increased $\mathrm{F}$ element ratio in the SEl layer. As shown in Figs. $1 \mathrm{j}$ and $4 \mathrm{~h}$, the added $\mathrm{F}$ element ratio in the SEl layer exists almost entirely in the form of LiF after the introduction of THE. After introducing THE, the increased ratio of F element is 0.29 for the $60 \%$ THE electrolyte. Based on Eq. 3, $\sigma_{2}$ is calculated to be $\sim 1.9 \times 10^{-7} \mathrm{~S} / \mathrm{cm}$, which is on the same order of that for $\mathrm{LiF}\left(\sigma_{\mathrm{LiF}}, 6.4 \times 10^{-7} \mathrm{~S} / \mathrm{cm}^{2}\right)$ and far beyond that of conventional electrolyte/anode interface $\left(\sigma_{1}, 1 \times 10^{-9} \mathrm{~S} / \mathrm{cm}^{2}\right){ }^{26,31}$ Such an enhancement on the ionic conductivity at electrolyte/anode interface gives rise to the improved rate performances with the $60 \%$ THE electrolyte. It is noted that regardless of the anode material the SEI film is mainly formed through the reaction of the deposited Li with the electrolyte. ${ }^{32}$ Therefore, the THE electrolyte improves the ionic conductivity of SEI and rate performances of lithium ion batteries in general.

The mechanism of C-F bond cleavage is further studied. Owing to a low LUMO energy value (Fig. 1e), THE on the anode surface is prone to defluorination through reduction reaction. AIMD simulations (Fig. S15, Supporting Information) show the $\mathrm{C}-\mathrm{F}$ bond breaks on the $\mathrm{C}$ atom between the $\mathrm{CF}_{2}$ and the $\mathrm{CF}_{3}$ groups of THE. As shown in Fig. 4g, the energy barriers for the C-F bond cleavage of TTEE and 2,2,2trifluoroethyl-1,1,2-trifluoropropyl ether (TTRE) are $0.69 \mathrm{eV}$ and $0.14 \mathrm{eV}$, considerably above that of THE $(0.04 \mathrm{eV})$, indicating that the efficient formation of LiF through C-F bond cleavage with the THE electrolyte. $^{33}$

The energy barrier for $\mathrm{Li}^{+}$mobility at the deposited $\mathrm{Li}\left(\begin{array}{lll}0 & 0 & 1\end{array}\right)$ /electrolyte interface is calculated through AIMD simulations (Figs. 4i and S16, Supporting Information). For the THE-based electrolyte, the energy barrier is 1/7 and 1/6 those of the convential DME and DOL electrolytes, since THE is a free solvent molecule and $\mathrm{Li}^{+}$does not completely strip off the solvent molecules before intercalation into the anode, 
interface. AIMD calculations (Fig. 4h) show that F transfers from the THE solvent to the deposited Li surface, resulting in LiF-rich inorganic species near the anode surface, as observed with in-situ Raman (Figs. $1 \mathrm{l}$ and $1 \mathrm{~m}$ ). The LiF-rich interphase layers on the electrodes improve the reaction kinetics and cycle stability of the batteries.

\section{Conclusion}

In summary, THE-based electrolyte is first developed by employing fluoroalkyl ether THE as the cosolvent in LiTFSI/DOL + DME. Both experiments and computational simulations demonstrate that the THE-based electrolyte with interface ion affinity owns excellent wettability to the commercial separator, rapid and uniform $\mathrm{Li}^{+}$transmission, and promotes the formation of LiF-rich SEl with mitigated Li dendrites. Using the $60 \% \mathrm{THE}$ electrolyte, the $\mathrm{LiFePO}_{4}$ cathode exhibits sustainably enhanced cycling stability and unparalleled low capacity fading in high-rate long cycle. This study offers a promising strategy to enable THE-based electrolytes with rapid interface ion transference for high-performance energy-storage devices.

\section{References}

1. Griffith, K. J., Wiaderek, K. M., Cibin, G., Marbella, L. E. \& Grey, C. P. Niobium tungsten oxides for highrate lithium-ion energy storage. Nature 559, 556-563 (2018).

2. Peters, B. K. et al. Scalable and safe synthetic organic electroreduction inspired by Li-ion battery chemistry. Science 363, 838-845 (2019).

3. Liu, J. et al. Metal oxides with distinctive valence states in an electron-rich matrix enable stable highcapacity anodes for Li ion batteries. Small Methods 4, 190053 (2019).

4. Liu, Q. et al. Approaching the capacity limit of lithium cobalt oxide in lithium ion batteries via lanthanum and aluminium doping. Nat. Energy 3, 936-943 (2018).

5. He, M. et al. High voltage LiNi $0.5 \mathrm{Mn}_{0.3} \mathrm{Co}_{0.2} \mathrm{O}_{2}$ /graphite cell cycled at $4.6 \mathrm{~V}$ with a FEC/HFDEC-based electrolyte. Adv. Energy Mater. 7, 1700109 (2017).

6. Chen, S. et al. Self-supported $\mathrm{Li}_{4} \mathrm{Ti}_{5} \mathrm{O}_{12}$ nanosheet arrays for lithium ion batteries with excellent rate capability and ultralong cycle life. Energy Environ. Sci. 7, 1924-1930 (2014).

7. Billaud, J., Bouville, F., Magrini, T., Villevieille, C. \& Studart, A. R. Magnetically aligned graphite electrodes for high-rate performance Li-ion batteries. Nat. Energy 1, 16097 (2016).

8. Xue, W. et al. Intercalation-conversion hybrid cathodes enabling Li-S full-cell architectures with jointly superior gravimetric and volumetric energy densities. Nat. Energy 4, 374-382 (2019).

9. Liu, J. et al. Pathways for practical high-energy long-cycling lithium metal batteries. Nat. Energy 4, 180-186 (2019).

10. Zhang, C. et al. Anion-sorbent composite separators for high-rate lithium-ion batteries. Adv. Mater. 31, 1808338 (2019). 
11. Graves, C., Ebbesen, S. D., Jensen, S. H., Simonsen, S. B. \& Mogensen, M. B. Eliminating degradation in solid oxide electrochemical cells by reversible operation. Nat. Mater. 14, 239-244 (2014).

12. Gao, Y. et al. Polymer-inorganic solid-electrolyte interphase for stable lithium metal batteries under lean electrolyte conditions. Nat. Mater. 18, 384-389 (2019).

13. Xu, K. Nonaqueous liquid electrolytes for lithium-based rechargeable batteries. Chem. Rev. 104, 4303-4417 (2004).

14. Jiao, S. et al. Stable cycling of high-voltage lithium metal batteries in ether electrolytes. Nat. Energy $\mathbf{3}$, 739-746 (2018).

15. Liu, W. et al. Enhancing ionic conductivity in composite polymer electrolytes with well-aligned ceramic nanowires. Nat. Energy 2, 17035 (2017).

16. Zhao, Q., Liu, X., Stalin, S., Khan, K. \& Archer, L. A. Solid-state polymer electrolytes with in-built fast interfacial transport for secondary lithium batteries. Nat. Energy 4, 365-373 (2019).

17. Elia, G. A., Ulissi, U., Jeong, S., Passerini, S. \& Hassoun, J. Exceptional long-life performance of lithium-ion batteries using ionic liquid-based electrolytes. Energy Environ. Sci. 9, 3210-3220 (2016).

18. Yang, C. et al. Aqueous Li-ion battery enabled by halogen conversion-intercalation chemistry in graphite. Nature 569, 245-250 (2019).

19. Yamada, Y. et al. Unusual stability of acetonitrile-based superconcentrated electrolytes for fastcharging lithium-ion batteries. J. Am. Chem. Soc. 136, 5039-5046 (2014).

20. Liao, B. et al. Designing low impedance interface films simultaneously on anode and cathode for high energy batteries. Adv. Energy Mater. 8, 1800802 (2018).

21. Conder, J. et al. Direct observation of lithium polysulfides in lithium-sulfur batteries using operando X-ray diffraction. Nat. Energy 2, 17069 (2017).

22. Wang, J. et al. Fire-extinguishing organic electrolytes for safe batteries. Nat. Energy 3, 22-29 (2018).

23. Suo, L. et al. "Water-in-salt” electrolyte enables high-voltage aqueous lithium-ion chemistries. Science 350, 938-943 (2015)

24. Qian. J. et al. High rate and stable cycling of lithium metal anode. Nat. Commun. 6, 6362 (2015)

25. Chen, K.-H. et al. Dead lithium: mass transport effects on voltage, capacity, and failure of lithium metal anodes. J. Mater. Chem. A 5, 11671-11681 (2017).

26. Li, C., Gu, L., Guo, X., Samuelis, D., Tang, K. \& Maier, J. Charge carrier accumulation in lithium fluoride thin films due to Li-ion absorption by titania (100) subsurface. Nano. Lett. 12, 1241-1246 (2012).

27. Lu, Y., Tu, Z. \& Archer, L. A. Stable lithium electrodeposition in liquid and nanoporous solid electrolytes. Nat. Mater. 13, 961-969 (2014).

28. Zheng, J. et al. High-fluorinated electrolytes for Li-S batteries. Adv. Energy Mater. 9, 1803774 (2019).

29. Zu, C., Azimi, N., Zhang, Z. \& Manthiram, A. Insight into lithium-metal anodes in lithium-sulfur batteries with a fluorinated ether electrolyte. J. Mater. Chem. A 3, 14864-14870 (2015).

30. Chen, W. et al. Designing safe electrolyte systems for a high-stability lithium-sulfur battery. Adv. 
31. Han, B. et al. Self-regulated phenomenon of inorganic artificial solid electrolyte interphase for lithium metal batteries. Nano. Lett. 20, 4029-4037 (2020).

32. Ding, J. Hu, W., Paek, E. \& Mitlin, D. Review of Hybrid Ion Capacitors: From Aqueous to Lithium to Sodium. Chem. Rev. 118, 6457-6498 (2018).

33. Amanchukwu, C. Harding, J., Shao, Y. \& Hammond, P. Understanding the chemical stability of polymers for lithium-air batteries. Chem. Mater. 27, 550-561 (2015).

\section{Figures}
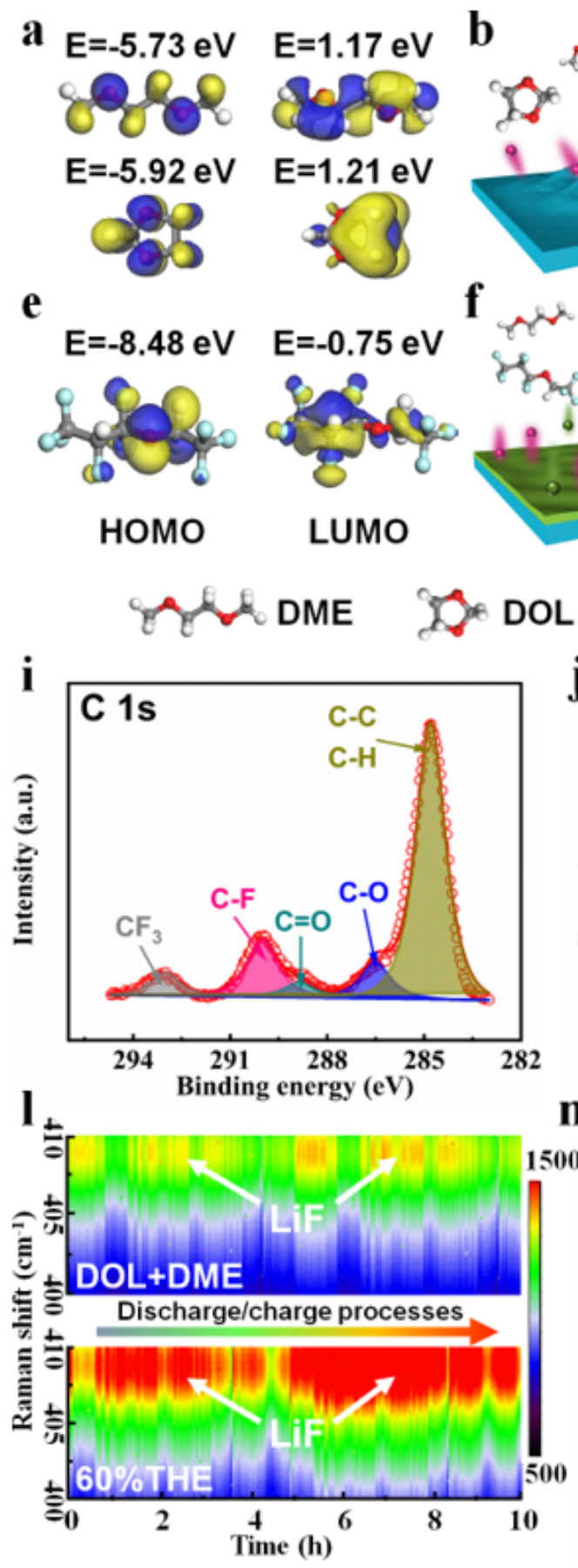

\section{m}
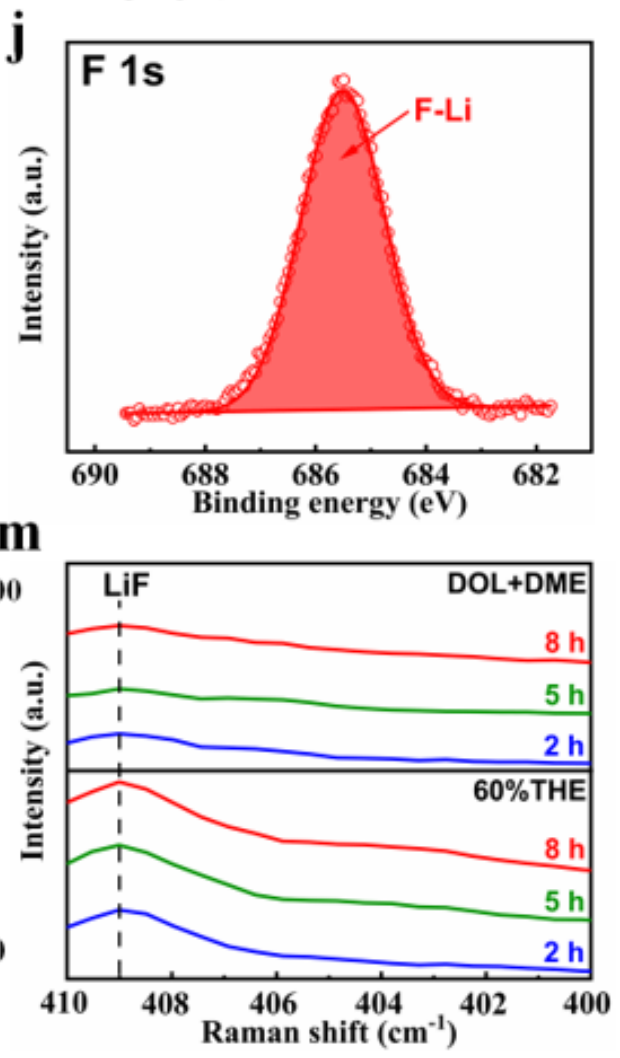

Anode
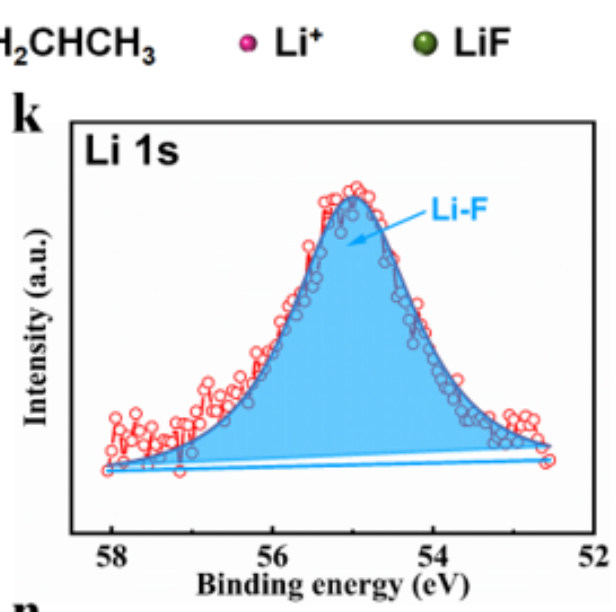

n

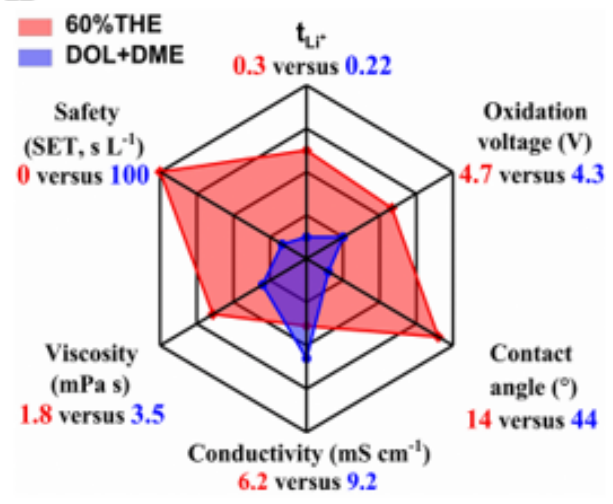


Figure 1

HOMO and LUMO values of a, DME, DOL, and e, THE. Schematics of anode surface and Li+ transmission in b, DOL+DME and $\mathrm{f}, 60 \%$ THE electrolytes. Titled SEM and cross-sectional SEM images of Li anodes in c,d, DOL+DME and g,h, 60\%THE electrolytes after 100 cycles, yellow lines show the etching depth. i-k, XPS of the SEl layer in the $60 \%$ THE electrolyte, C 1s, F 1s, and Li 1s spectra are presented, including peak deconvolution and assignments. I, In-situ Raman images of DOL+DME and 60\%THE electrolytes in discharge/charge. m, Selected Raman spectroscopies of DOL+DME and 60\%THE electrolytes. n, Comparison of the properties of conventional DOL+DME and 60\%THE electrolytes.

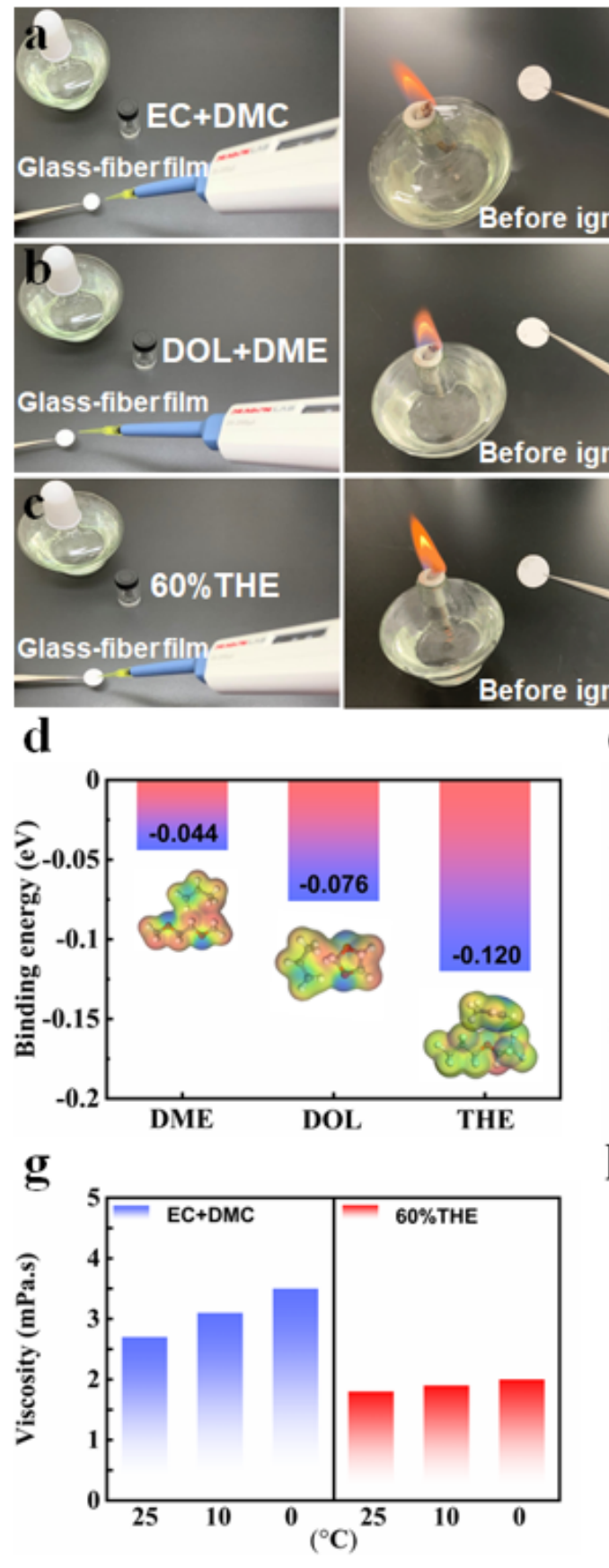

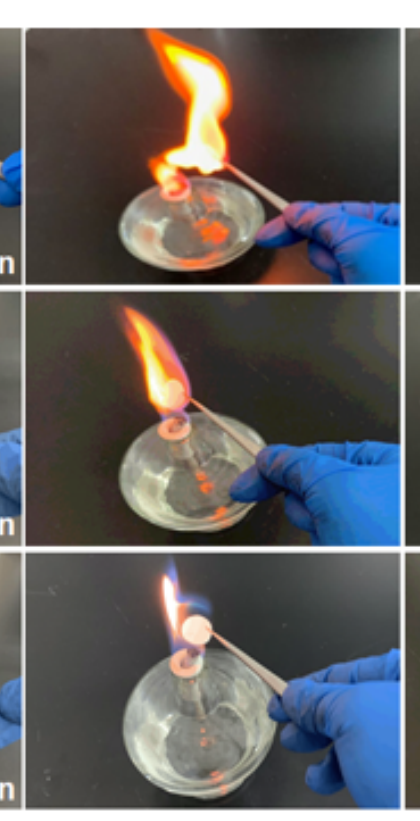

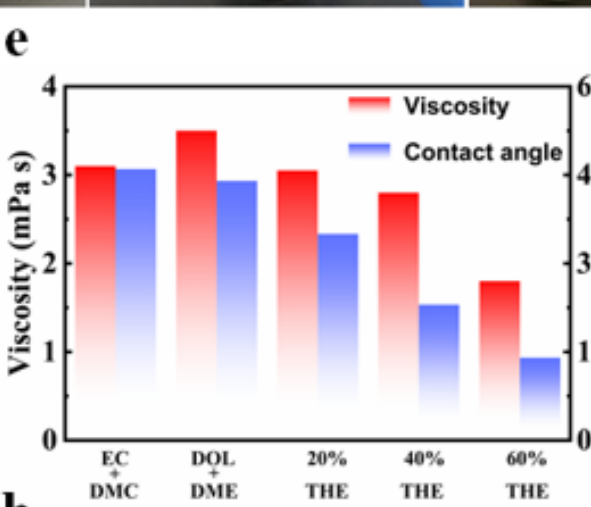

h

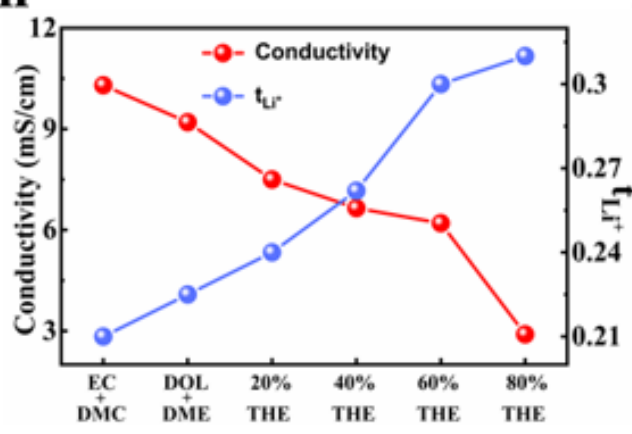

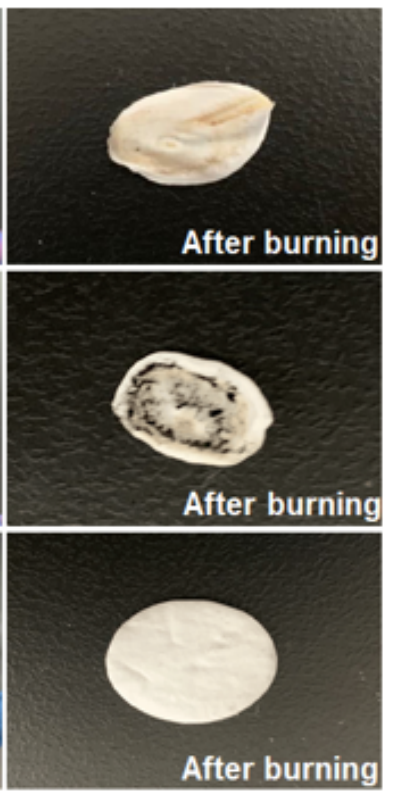

After burning
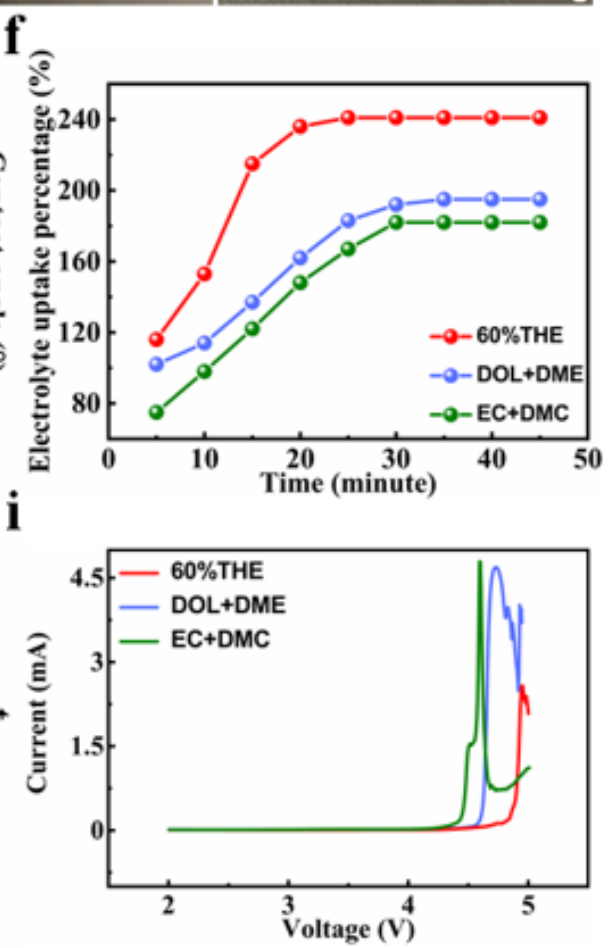

\section{Figure 2}


Ignition tests for a, EC+DMC, b, DOL+DME, and c, 60\%THE electrolytes on glass-fiber films. d, Adsorption energy and corresponding electron density maps for $\mathrm{CH} 2 \mathrm{CHCH} 3$ with $\mathrm{DME}, \mathrm{DOL}$, and THE. e, Viscosity and contact angle analysis for various electrolytes. $\mathrm{f}$, Uptake curves of different electrolytes over time. $\mathrm{g}$, Viscosity of EC+DMC and $60 \%$ THE electrolytes with temperatures. h, Conductivity and t_( $\left(\mathrm{Li}^{\wedge}+\right)$ with different electrolytes. i, Electrochemical windows of EC+DMC, DOL+DME and 60\%THE electrolytes.

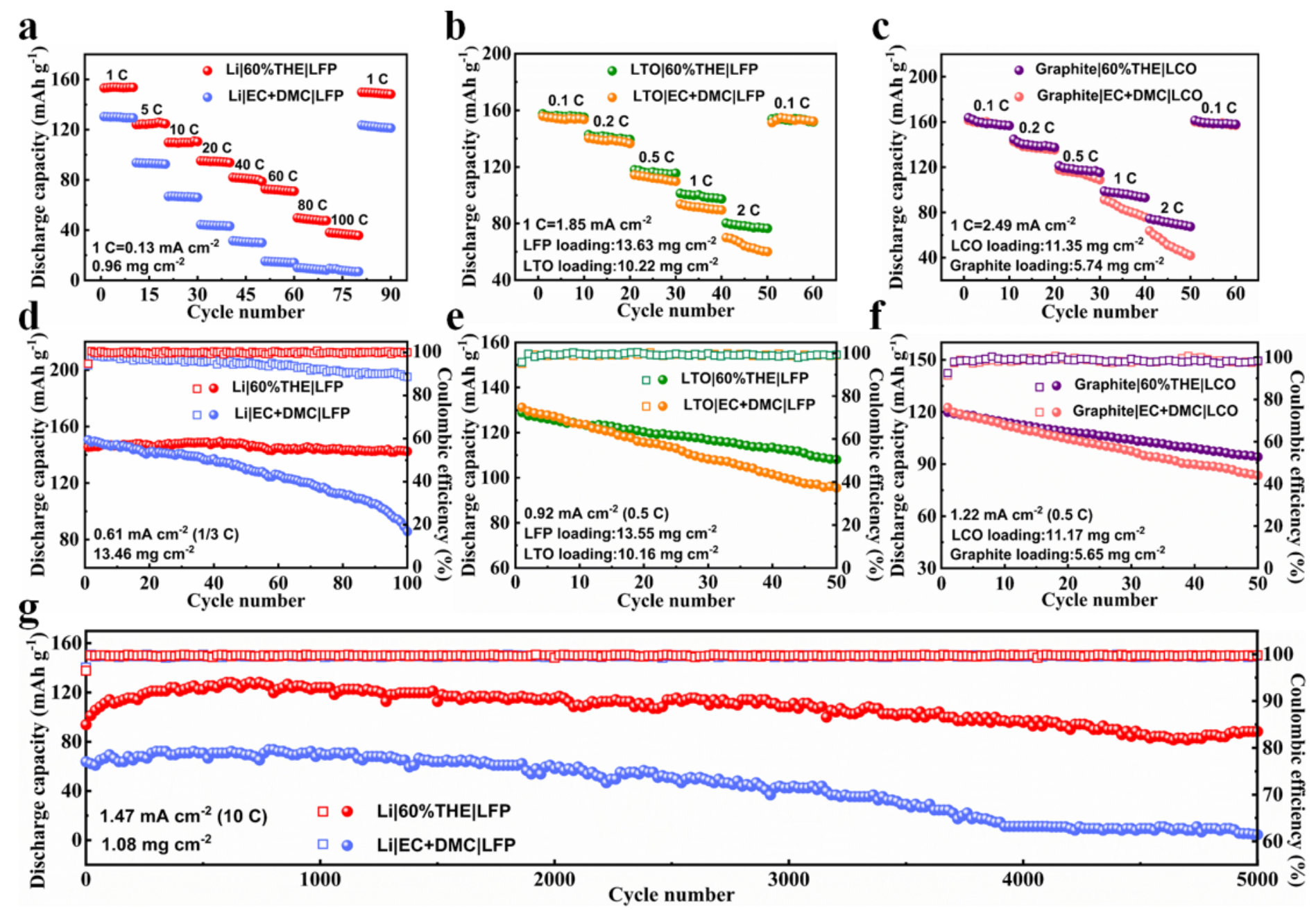

Figure 3

Rate performances of the $a$, Li/LFP, b, LTO/LFP, and c, graphite/LCO cells with EC+DMC and $60 \%$ THE electrolytes. Cycling performances of the $\mathrm{d}$, Li/LFP (1/3 C), e, LTO/LFP (1/2 C), and f, graphite/LCO (1/2 C) batteries with EC+DMC and $60 \%$ THE electrolytes at high-loading. g, Long-term cycling performances of the Li/LFP cells with EC+DMC and 60\%THE electrolytes at $10 \mathrm{C}$. 
a

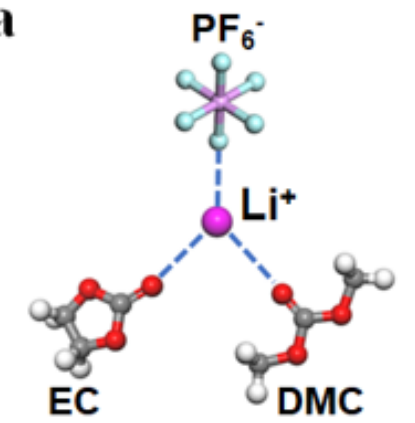

d

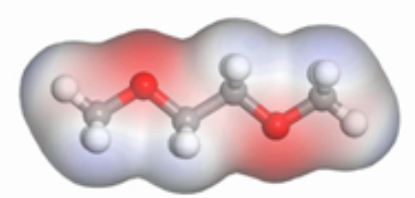

g

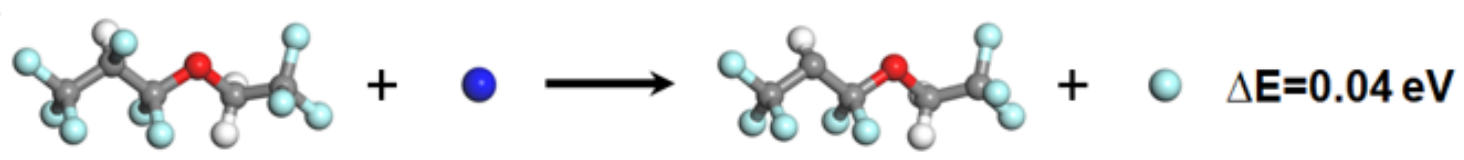

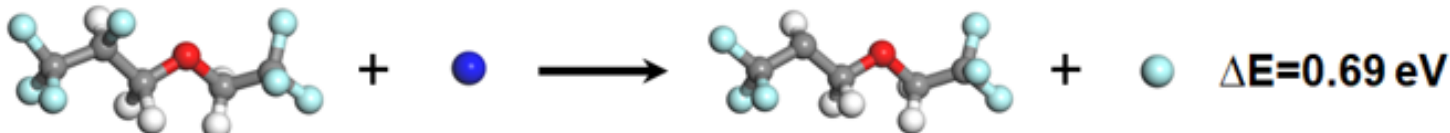
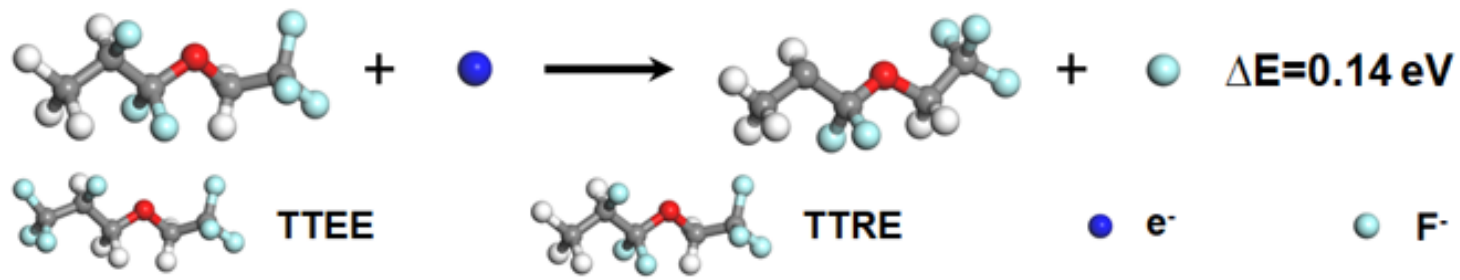

$\mathbf{h}$

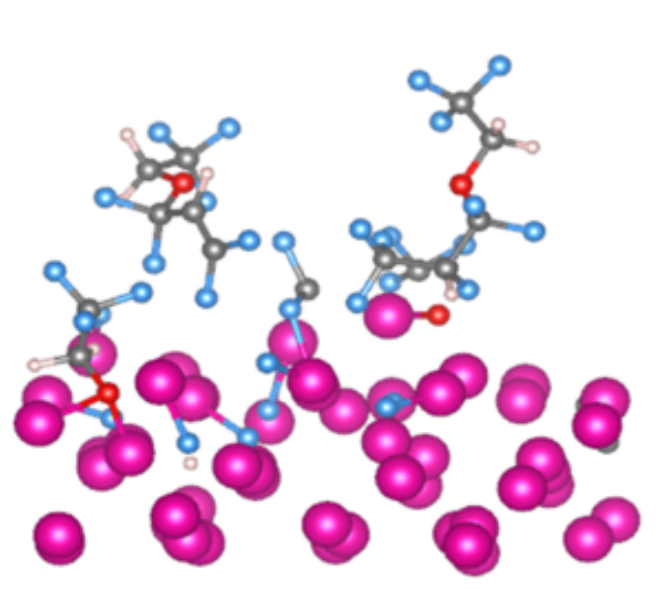

c

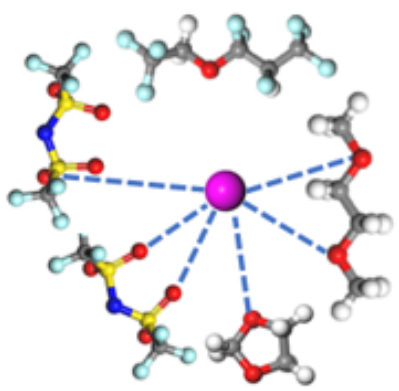

$\mathbf{e}$

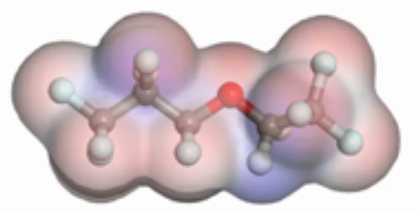

f

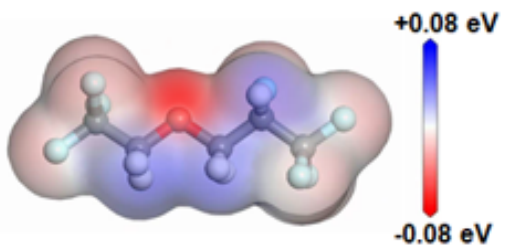

- $\mathbf{e}^{-} \quad \mathrm{F}^{-}$

i

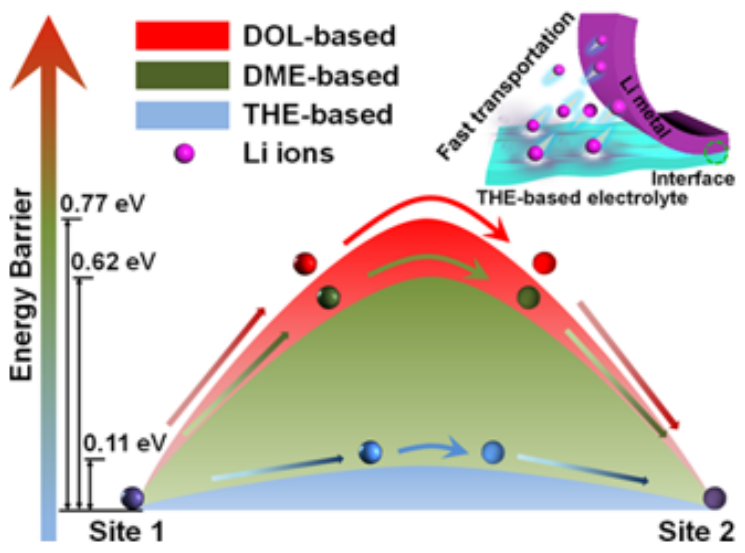

Figure 4

The representative Li+ solvation structures of (a) EC/DMC, (b) DOL/DME, and (c) 60\%THE electrolytes respectively. ESP comparison of (d) DME, (e) THE, and (f) TTEE. $g$, The energy barriers for the C-F bond cleavage of THE, TTEE (CF2 is replaced $\mathrm{CH} 2$ ) and TTRE (CF3 is replaced $\mathrm{CH} 3$ ). h, Adsorption of fluorinerich THE on deposited Li $\left(\begin{array}{lll}0 & 0 & 1\end{array}\right)$ surface accompanied with abundant LiF formation. i, Energy barriers for 
Li transfer in DOL, DME, and THE solvents to deposited Li $\left(\begin{array}{lll}0 & 0 & 1\end{array}\right)$ surface. Balls with various colors represent different atoms; color code: red, O; blue, N; yellow, S; blue, F; magenta, Li; grey, C; white H.

\section{Supplementary Files}

This is a list of supplementary files associated with this preprint. Click to download.

- VideoS1.TheignitionexperimentofECDMCelectrolyte.mp4

- VideoS2.TheignitionexperimentofDOLDMEelectrolyte.mp4

- VideoS3.Theignitionexperimentof60XTHEelectrolyte.mp4

- Supplementarylnformation.docx 